\title{
BMJ Open Safety of tranexamic acid in thrombotic adverse events and seizure in patients with haemorrhage: a protocol for a systematic review and meta-analysis
}

To cite: Murao S, Nakata H, Yamakawa K. Safety of tranexamic acid in thrombotic adverse events and seizure in patients with haemorrhage: a protocol for a systematic review and meta-analysis. BMJ Open 2020;10:e036020. doi:10.1136/ bmjopen-2019-036020

- Prepublication history and additional material for this paper are available online. To view these files, please visit the journal online (http://dx.doi org/10.1136/bmjopen-2019036020).

Received 26 November 2019 Revised 08 May 2020 Accepted 29 May 2020

\section{Check for updates}

(c) Author(s) (or their employer(s)) 2020. Re-use permitted under CC BY-NC. No commercial re-use. See rights and permissions. Published by BMJ.

Division of Trauma and Surgical Critical Care, Osaka General Medical Center, Osaka, Japan

Correspondence to

Dr Kazuma Yamakawa;

k.yamakawa0911@gmail.com

\section{ABSTRACT}

Introduction Tranexamic acid (TXA) is a synthetic derivative of the amino acid lysine that inhibits fibrinolysis by blocking lysine-binding sites on plasminogen, which contribute to reduced bleeding, the need for transfusion and mortality. Although there is reliable evidence of the efficacy of TXA, its effects on other important outcomes, adverse events, including thrombotic events and seizure, remain uncertain.

Methods and analysis We will conduct a systematic review and meta-analysis of randomised controlled trials with the objective of evaluating the incidence of thrombotic adverse events and seizure and how the effect of TXA varies by dose and underlying disease. We will include patients with bleeding in any underlying disease. We will search MEDLINE, EMBASE and Cochrane Central Register of Controlled Trials for randomised controlled trials. The planned date of our systematic search is 1 June 2020. We will follow the recommendations of the Cochrane Collaboration and the Preferred Reporting Items for Systematic Review and Meta-Analysis statement. Subgroup and sensitivity analyses will be performed to explore residual heterogeneity and inconsistency. Metaregression analysis will be carried out to investigate the association between the incidence of adverse events and the TXA dose. The risk of systematic errors (bias) and random errors will be assessed and the overall quality of evidence will be evaluated using the Grading of Recommendations Assessment, Development and Evaluation approach.

Ethics and dissemination This study will not involve primary data collection, and formal ethics approval will therefore not be required. We aim to publish this systematic review in a peer-review journal.

Trial registration number UMIN000039611.

\section{INTRODUCTION}

Tranexamic acid (TXA) is a long-established antifibrinolytic agent that was developed in Japan in $1965 .{ }^{12}$ TXA is a synthetic derivative of the amino acid lysine that inhibits fibrinolysis by blocking the lysine-binding sites on plasminogen, which contribute to reduced bleeding. ${ }^{3}$ For many years, TXA has been used in the surgical field to reduce blood loss and the need for transfusion. ${ }^{4}$ Recent large-scale

\section{Strengths and limitations of this study}

- This study will be the first systematic review and meta-analysis focusing on the safety of tranexamic acid (TXA) in thrombotic adverse events and seizure in patients with haemorrhage.

- We will integrate all of the available evidence from randomised controlled trials regardless of the underlying disease, which will increase the pooled population size and become clinically useful data in patients with haemorrhage overall.

- We will also perform meta-regression analysis to assess how the incidence of adverse events varies by TXA dosage.

- The quality of evidence will be evaluated using the Grading of Recommendations Assessment, Development and Evaluation system.

- Given that TXA has received considerable attention in various clinical situations, clinicians all over the world are concerned whether TXA can be used safely or should be used cautiously.

randomised controlled trials (RCTs) have shown that early administration of TXA reduced death from bleeding in patients with trauma and postpartum haemorrhage,$^{56}$ and its use is still expanding to include spontaneous intracranial bleeding and gastrointestinal bleeding. ${ }^{78}$

Although there is reliable evidence of the efficacy of TXA, its effects on other important outcomes, adverse events, including thrombotic adverse events and seizure, have not been well investigated in patients with haemorrhage overall. TXA theoretically has prothrombotic properties, whereas no increased risk of thromboembolism was observed in patients with trauma, postpartum haemorrhage and cardiac surgery. ${ }^{5} \quad 6 \quad 9-13$ Despite this cumulative evidence, uncertainties remain due to an inadequate amount of information on thrombotic adverse events, ${ }^{4}$ and the risk of TXA-associated thrombotic complication is still a concern for clinicians. 
As a lysine analogue, TXA can cross the blood-brain barrier and thus may act on neurons and induce disorders of the brain. ${ }^{14}{ }^{15}$ In fact, some studies have indicated that high-dose TXA was associated with an increased risk of seizure in patients with cardiac diseases, ${ }^{13}{ }^{16-18}$ among whom renal dysfunction is the major risk factor for seizure due to elevated plasma TXA concentration. ${ }^{19} 20$

However, high certainty of evidence focusing on the incidence of adverse events and its relationship with the TXA dosage is still lacking. We hypothesised that harm caused by TXA should not differ between underlying haemorrhagic diseases. The aim of this systematic review and meta-analysis is to assess the incidence of adverse events in patients with haemorrhage allocated to TXA versus placebo or no treatment. We will also evaluate how the effect of TXA varies by dose and underlying disease with subanalysis and meta-regression analysis.

\section{METHODS}

\section{Protocol}

This study adopts recommendations on the conduct and reporting of systematic reviews and meta-analyses outlined by the Preferred Reporting Items for Systematic Reviews and Meta-Analysis Protocol (PRISMA-P) checklist (online supplementary file 1). ${ }^{21}{ }^{22}$ The protocol was developed according to the PRISMA-P. ${ }^{23}$ The review has been registered in the University Hospital Medical Information Network (https://www.umin.ac.jp/english/).

\section{Focused review questions}

The objective of this review is to evaluate the effect of TXA on the incidence of adverse events, including thrombotic events and seizure, in patients with haemorrhage.

\section{Types of studies}

We will include only RCTs that studied TXA in patients with bleeding.

\section{Condition or domain being studied}

The study domain will include adverse events reported as part of an RCT.

\section{Types of participants}

We will include patients administered TXA for any underlying disease such as trauma, surgery, postpartum haemorrhage, spontaneous intracranial haemorrhage and gastrointestinal haemorrhage. Studies done with animals will be excluded from this review.

\section{Types of interventions and comparators}

We will include studies that describe the administration of any amount of TXA, whether by intravenous bolus and/ or infusion, to control haemorrhage for any underlying disease. The comparator or control group must include patients who received a placebo or no treatment.

\section{Outcomes}

The primary outcome measures will be the number of patients with any thrombotic adverse events, including acute coronary syndrome (ACS), stroke/transient ischaemic attack (TIA) and venous thromboembolism (VTE), and seizure at the most protracted time reported in clinical trials. As secondary outcome measures, the number of patients with ACS, stroke/TIA and VTE will be assessed separately.

\section{Search strategy}

We will search the following databases for relevant studies: MEDLINE (via PubMed), EMBASE and the Cochrane Central Register of Controlled Trials. We will not apply any language or time restrictions on electronic searches. The search strategy in MEDLINE is as follows: ((Tranexamic acid [mesh] OR tranexamic acid [tiab] OR TXA [tiab]) AND ((randomized controlled trial [pt] OR controlled clinical trial [pt] OR randomized [tiab] OR clinical trials as topic [mesh: noexp] OR randomly [tiab] OR trial [ti]) NOT (animals [mh] NOT humans $[\mathrm{mh}]$ ). Our MEDLINE search strategy will be adapted for searches in the other two databases. The planned date of our systematic search is 1 June 2020.

\section{Citation management and screening}

Citations will be stored and duplicates will be removed using EndNote software (Thomson Reuters, Toronto, Ontario, Canada). Studies will be screened initially according to title and abstract by two authors ( $\mathrm{HN}$ and SM) independently and those not meeting the criteria will be discarded. Disagreements will be resolved by discussion and referral to a third author $(\mathrm{KY})$ if necessary. After this initial stage, the full text of all remaining studies will be reviewed by two authors ( $\mathrm{HN}$ and $\mathrm{SM}$ ) independently for inclusion or exclusion in the final study. Again, disagreements will be resolved by discussion and referral to a third author $(\mathrm{KY})$ if necessary. We will document the study selection process in a PRISMA flow diagram.

\section{Data abstraction}

Two authors (HN, SM) will extract the study characteristics from each included study, including year of publications, study population, number of participants, name of comparators, dose and duration of treatment, indication for treatment, name and types of adverse events, and information assessing the risk of bias in the studies. Adjudication by a third author (KY) will be used if necessary. Efforts will be made to contact the authors of the primary studies to provide missing data where necessary.

\section{Assessment of the risk of bias}

Two authors (HN and SM), who are not involved in any of the included trials and are blinded to the results of the meta-analysis, will independently assess the risk of systematic errors (bias) of the trials included in the meta-analysis according to the Cochrane Handbook, version 5.1.0. ${ }^{14}$ Disagreements during the review process will be resolved by consensus through the involvement of a third review author (KY). The risk of bias will be rated according to the following domains (1) sequence generation, (2) allocation concealment, (3) blinding 
of participants and personnel, (4) blinding of outcome assessment, (5) incomplete outcome data, (6) selective outcome reporting and (7) other sources of bias (specifically including baseline imbalance, early stopping and financial bias). Trials adjudicated as unclear or having a high risk of bias for one or more domains will be classified as having an overall high risk of bias.

\section{Rating of the certainty of evidence using the grading of recommendations, developments and evaluation approach}

The risk of systematic errors (bias) and random errors will be assessed, and the overall quality of evidence will be evaluated using the Grading of Recommendations Assessment, Development and Evaluation (GRADE) approach.

\section{Data synthesis}

Statistical analyses will be performed using Review Manager Software V.5 (Review Manager (RevMan) V.5.3; Copenhagen: The Nordic Cochrane Centre, The Cochrane Collaboration, 2014) and STATA software, V.14.0 (STATA Corporation). For each included trial, we will calculate the relative risks with $95 \%$ CIs for all outcome measures. Heterogeneity among the trials will be explored by inspection of forest plots and calculation of $\mathrm{I}^{2}$ statistics. ${ }^{24}$ We will perform both fixed-effects analysis using the Mantel-Haenszel method and random-effects analyses using the DerSimonian and Laird estimator, reporting the most conservative summary estimate with the broadest CI. ${ }^{25}$ Subgroup analyses will be performed as standard using RevMan V.5 software.

\section{Assessment of heterogeneity}

We will investigate heterogeneity initially by visual examination of forest plots. Statistical heterogeneity will be evaluated informally from forest plots of the study estimates and more formally using the $\chi^{2}$ test ( $p$ value $<0.1=$ significant heterogeneity) and $\mathrm{I}^{2}$ statistic $\left(\mathrm{I}^{2}>50 \%=\right.$ significant heterogeneity).

\section{Assessment of publication biases}

If a sufficient number of studies are identified, then we will investigate publication biases by the Deek funnel plot. We will interpret this analysis with caution given the lack of statistical power of this test and the absence of consensus about adequate methods to detect publication bias in reviews of diagnostic test accuracy.

\section{Sensitivity and subgroup analysis}

We will examine the robustness of the meta-analyses by conducting sensitivity analyses and excluding studies according to different components of the GRADE tool for assessing the risk of bias. Our primary analysis will include all studies, whereas the sensitivity analysis will exclude studies with a high risk of bias or with important concerns about potential applicability. If sufficient studies are available, we will undertake the following subgroup analyses: year of publication, country, sample size $(<500$ or $\geq 500$ ), dose of TXA ( $\leq 2$ or $>2 \mathrm{~g} /$ day) and type of underlying disease (trauma, neurosurgery, cardiac surgery, orthopaedic surgery, obstetrics and gynaecology, gastrointestinal bleeding and others). Meta-regression analysis will be carried out to investigate the association between the incidence of adverse events and the dose of TXA.

\section{Patient and public involvement}

No patients are involved.

\section{Ethics and dissemination}

This study will not involve primary data collection and formal ethics approval will therefore not be required. We aim to publish this systematic review in a peer-review journal.

\section{DISCUSSION}

TXA is commonly used to reduce blood loss in traumatic and surgical settings. Many clinical trials have shown the efficacy of TXA and its use is still expanding. ${ }^{78}$ Although there is reliable evidence of the efficacy of TXA, its safety has not been adequately assessed. Considering the fact that the observed risk reduction on mortality was small in the TXA groups, ${ }^{5-7}$ the incidence of adverse events will be of great concern to clinicians when deciding on TXA use. From the perspective of the basic mechanism of TXA, its shutdown of fibrinolysis potentially increases the risk of thrombotic events ${ }^{10-12} 26$ although we found no increase in recently published RCTs. ${ }^{5-7} 27$ Under experimental conditions, TXA induces convulsive seizure when administered intrathecally, ${ }^{28}$ and indeed, some observational studies and an RCT indicated that the administration of high-dose TXA was associated with increased risk of seizure. ${ }^{13} 16-18$

However, the overall impact of TXA on the incidence of these adverse events and its relationship with dose remain uncertain. Based on the assumption that the harm due to the TXA administration should not differ from that caused by the underlying disease, we will integrate all of the available evidence from the RCTs, regardless of the underlying disease. This systematic review and meta-analysis will provide clinicians with a clearer understanding of the strength and limitations of TXA administration.

We acknowledge potential limitations. First, we will include all patients with haemorrhage. Although this patient selection will contribute to increasing the pooled sample size, it might introduce clinical heterogeneity. However, we will conduct a detailed preplanned subgroup analysis according to underlying diseases to evaluate heterogeneity between different disease types and administration statuses. Second, there could be reporting bias as inadequate reporting of adverse events is not rare in clinical trials. Our preliminary search confirmed that recent large-scale RCTs included major potential adverse events involved in this review.

Contributors KY contributed to the conception of the study. The manuscript protocol was drafted by SM and revised by KY. The search strategy was developed by all authors and will be performed by SM and HN, who will also independently screen the potential studies, extract data from the included studies, assess the risk 
of bias and complete the data synthesis. KY will arbitrate in cases of disagreement and ensure the absence of errors. All authors approved the publication of this protocol.

Funding This systematic review received funding from ZENKYOREN (National Mutual Insurance Federation of Agricultural Cooperatives). No funding body had any role in developing the protocol of this systematic review and meta-analysis.

Competing interests None declared.

Patient and public involvement Patients and/or the public were not involved in the design, or conduct, or reporting, or dissemination plans of this research.

Patient consent for publication Not required.

Provenance and peer review Not commissioned; externally peer reviewed.

Open access This is an open access article distributed in accordance with the Creative Commons Attribution Non Commercial (CC BY-NC 4.0) license, which permits others to distribute, remix, adapt, build upon this work non-commercially, and license their derivative works on different terms, provided the original work is properly cited, appropriate credit is given, any changes made indicated, and the use is non-commercial. See: http://creativecommons.org/licenses/by-nc/4.0/.

ORCID iD

Kazuma Yamakawa http://orcid.org/0000-0003-2999-4021

\section{REFERENCES}

1 Okamoto S, Okamoto U. Amino-methyl-cyclohexane-carboxylic acid: AMCHA. A new potent inhibitor of the fibrinolysis. Keio $\mathrm{J}$ Med 1962;11:105-15.

2 Kobayashi T, Sugiura J. The effect of a new potent antifibrinolytic agent, tranexamic acid. J Jpn Obstet Gynecol Soc 1966;13:158-67.

3 Okamoto S, Hijikata-Okunomiya A, Wanaka K, et al. Enzymecontrolling medicines: introduction. Semin Thromb Hemost 1997;23:493-501.

4 Ker K, Edwards P, Perel P, et al. Effect of tranexamic acid on surgical bleeding: systematic review and cumulative meta-analysis. BMJ 2012;344:e3054-66.

5 CRASH-2 trial collaborators, Shakur $\mathrm{H}$, Roberts I, et al. Effects of tranexamic acid on death, vascular occlusive events, and blood transfusion in trauma patients with significant haemorrhage (CRASH-2): a randomised, placebo-controlled trial. Lancet 2010;376:23-32.

6 WOMAN Trial Collaborators. Effect of early tranexamic acid administration on mortality, hysterectomy, and other morbidities in women with post-partum haemorrhage (woman): an international, randomised, double-blind, placebo-controlled trial. Lancet 2017;389:2105-16.

7 Sprigg N, Flaherty K, Appleton JP, et al. Tranexamic acid for hyperacute primary intracerebral haemorrhage ( $\mathrm{TICH}-2)$ : an international randomised, placebo-controlled, phase 3 superiority trial. Lancet 2018;391:2107-15.

8 Roberts I, Coats T, Edwards P, et al. HALT-IT--tranexamic acid for the treatment of gastrointestinal bleeding: study protocol for a randomised controlled trial. Trials 2014;15:450.
9 Coats T, Roberts I, Shakur H. Antifibrinolytic drugs for acute traumatic injury. Cochrane Database Syst Rev 2004;4:CD004896.

10 Moore EE, Moore HB, Gonzalez E, et al. Rationale for the selective administration of tranexamic acid to inhibit fibrinolysis in the severely injured patient. Transfusion 2016;56 Suppl 2:S110-4.

$11 \mathrm{Ng} \mathrm{W}$, Jerath A, Wąsowicz M. Tranexamic acid: a clinical review. Anaesthesiol Intensive Ther 2015;47:339-50.

12 Nishida T, Kinoshita T, Yamakawa K. Tranexamic acid and traumainduced coagulopathy. J Intensive Care 2017;5:5.

13 Myles PS, Smith JA, Forbes A, et al. Tranexamic acid in patients undergoing coronary-artery surgery. N Engl J Med 2017;376:136-48.

14 McCormack PL. Tranexamic acid: a review of its use in the treatment of hyperfibrinolysis. Drugs 2012;72:585-617.

15 Manji RA, Grocott HP, Leake J, et al. Seizures following cardiac surgery: the impact of tranexamic acid and other risk factors. Can J Anaesth 2012;59:6-13.

16 Kalavrouziotis D, Voisine P, Mohammadi S, et al. High-Dose tranexamic acid is an independent predictor of early seizure after cardiopulmonary bypass. Ann Thorac Surg 2012;93:148-54.

17 Murkin JM, Falter F, Granton J, et al. High-Dose tranexamic acid is associated with nonischemic clinical seizures in cardiac surgical patients. Anesth Analg 2010;110:350-3.

18 Sharma V, Katznelson R, Jerath $A$, et al. The association between tranexamic acid and convulsive seizures after cardiac surgery: a multivariate analysis in 11529 patients. Anaesthesia 2014;69:124-30.

19 Montes FR, Pardo DF, Carreño M, et al. Risk factors associated with postoperative seizures in patients undergoing cardiac surgery who received tranexamic acid: a case-control study. Ann Card Anaesth 2012;15:6-12.

20 Jerath A, Yang QJ, Pang KS, et al. Tranexamic acid dosing for cardiac surgical patients with chronic renal dysfunction: a new dosing regimen. Anesth Analg 2018;127:1323-32.

21 Moher D, Liberati A, Tetzlaff J, et al. Preferred reporting items for systematic reviews and meta-analyses: the PRISMA statement. Open Med 2009;3:e123-30.

22 Liberati A, Altman DG, Tetzlaff J, et al. The PRISMA statement for reporting systematic reviews and meta-analyses of studies that evaluate health care interventions: explanation and elaboration. $J$ Clin Epidemiol 2009;62:e1-34.

23 Moher D, Shamseer L, Clarke M, et al. Preferred reporting items for systematic review and meta-analysis protocols (PRISMA-P) 2015 statement. Syst Rev 2015;4:1.

24 Higgins J, Green S. Cochrane Handbook for systematic reviews of interventions. version 5.1.0. The Cochrane Collaboration, 2011. http://handbook.cochrane.org

25 Jakobsen JC, Wetterslev J, Winkel P, et al. Thresholds for statistical and clinical significance in systematic reviews with meta-analytic methods. BMC Med Res Methodol 2014;14:120.

26 Sperzel M, Huetter J. Evaluation of aprotinin and tranexamic acid in different in vitro and in vivo models of fibrinolysis, coagulation and thrombus formation. J Thromb Haemost 2007;5:2113-8.

27 CRASH-3 trial collaborators. Effects of tranexamic acid on death, disability, vascular occlusive events and other morbidities in patients with acute traumatic brain injury (CRASH-3): a randomised, placebocontrolled trial. Lancet 2019;394:1713-23.

28 Fodstad $\mathrm{H}$. Convulsive seizures following subdural application of fibrin sealant containing tranexamic acid in a rat model. Neurosurgery 2001;49:479-80. 